\title{
Impact of the Facial Attractiveness of a Social Reward on Event-Related Potential Activities and Task Performance
}

\author{
Yasuhiro Ogoshi, ${ }^{*}$ Sakiko Ogoshi, ${ }^{1}$ Tomohiro Takezawa, ${ }^{2}$ and Yoshinori Mitsuhashi ${ }^{3}$ \\ Graduate School of Engineering, University of Fukui, 3-9-1 Bunkyo, Fukui 910-8507, Japan \\ ${ }^{1}$ National Institute of Technology, Fukui College, Geshi-cho, Sabae-shi, Fukui 916-8507, Japan \\ ${ }^{2}$ The National Institute of Vocational Rehabilitation, 3-1-3 Wakaba, Mihama-ku, Chiba 261-0014, Japan \\ ${ }^{3}$ Faculty of Education and Regional Studies, University of Fukui, 3-9-1 Bunkyo, Fukui 910-8507, Japan
}

(Received October 30, 2015; accepted February 8, 2016)

Keywords: electroencephalography, contingent negative variation potentials, social reward, task performance

Male participants were presented with pictures of female faces and asked to evaluate the degree of attractiveness of each image using a questionnaire. Then, the impact of the degree of attractiveness on subsequent event-related potentials was determined using electroencephalography conducted while participants completed a word recognition memory task with the facial images presented as rewards for correct responses. The most attractive faces elicited the largest contingent negative variation potentials and the greatest improvements in performance.

\section{Introduction}

Human learning and cognitive function are considerably affected by environmental and emotional factors. People learn and develop their cognitive capacity through stimuli from the environment. People feel joy when learning has been emphasized and attributed to internal and external factors. Internal factors depend only on the needs and wants of the learner, whereas external factors depend on the motivation obtained from external sources. Therefore, to facilitate learning, it is important for the learner's environment to be appropriately arranged by others, by matching the learner's self-esteem with extraneous motivation. ${ }^{(1)}$ Rewards can be effective motivating tools. It is known that tangible rewards (such as money/valuables and drinks) can improve the performance of cognitive tasks. ${ }^{(2)}$ Furthermore, social rewards, such as people's smiles and attractive faces, particularly of the opposite sex, are also known to enhance cognitive task performance. ${ }^{(3)}$ Whether people find faces of the opposite sex attractive may depend on subjective factors inherent to the individual. The manner by which the attractiveness of a face affects cognitive functions, such as memory, is an important issue when considering subjective attractiveness as an indicator of the value of social rewards. By effectively using attractiveness as an index of reward value, its application in individualized learning, for example, using brainmachine interface (BMI) methods, can be predicted.

In this study, we administered a questionnaire before participants performed a memory task. Participants were asked to rate the face of a person of the opposite sex according to subjective attractiveness. Next, a memory task was presented on a computer display and participants indicated

"Corresponding author: e-mail: y-ogoshi@u-fukui.ac.jp 
recognition of previously presented words by pressing a button. If the response was correct, a facial image of the opposite sex was presented on the display as a reward. This was used to identify the differences in the effect of facial images of varying degrees of attractiveness that were provided as rewards for the memory task. We investigated how different degrees of attractiveness affected the participants' response accuracy in the memory task. We also recorded the event-related potentials (ERPs) of participants while performing the task to examine how contingent negative variations (CNVs) in brain waves associated with attention and anticipation immediately before reward presentation were affected by expectations of image attractiveness.

\section{Method}

\subsection{Participants}

All participants were male university students $(N=7$, mean age 22.25 years).

\subsection{Experimental conditions}

(1) Experimental environment

The laboratory used for the electroencephalography (EEG) recordings was a sealed room with dim illumination. The distance between the display and the participant's head was approximately $110 \mathrm{~cm}$. The viewing angles of the display were $11.4^{\circ}$ for facial image stimuli and $3.6^{\circ}$ for Japanese character stimuli.

(2) Stimuli for memorizing and reward

The stimuli for the memory task consisted of 900 words with two Japanese katakana characters having 30-289 meanings. ${ }^{(4)}$ Also, 15 female facial images were created by synthesizing facial images of celebrities posted on the Internet, using the facial image synthesizing software (FotoMorph), for presentation as rewards for correct responses in the memory task. Prior to the memory task, participants assessed the female facial images using a questionnaire and rated their attractiveness. We had each subject complete the questionnaire presented in Fig. 1 for each of 15 photographs of women's faces prior to the experiment. The first question asked respondents to rate the attractiveness of the woman in the photograph on a Likert scale with highly attractive being assigned a value of 10 , moderately attractive a value of 0 and unattractive a value of -10 . Other questions on the questionnaire were not used in the selection of the image used in the experiment.

These ratings were used as an indicator of the attractiveness of the faces that were used. We specifically selected images with high and low attractiveness and used them as social rewards. We also created a square image painted on a gray background for presentation as a non-reward for incorrect responses. All images were adjusted so that they had approximately the same brightness.

(3) Measures

The following devices were used for EEG recording: (1) polymate AP1532 biological amplifier (TEAC) and (2) $\mathrm{Ag} / \mathrm{AgCl}$ plate electrodes with an internal diameter of $7 \mathrm{~mm}$ (NE-113A, Nihon Kohden). 


\section{Questionnaire}

\section{Age: [ ]years, [ ]months old, Sex:}

Please reply to the following items to see the photograph of the face of the next woman. Please circle the numerical value that best suits your response.

No.1

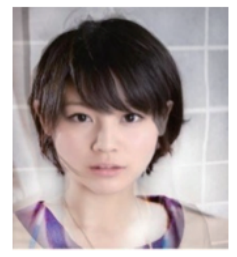

(1) How attractive do you feel the woman's face is in the photograph? Not at all attractive Slightly attractive Moderately attractive Very attractive Extremely attractive

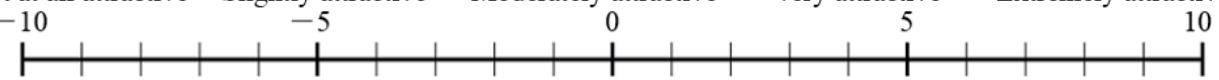

(2) Do you feel that the photograph of the face of this woman is pretty?

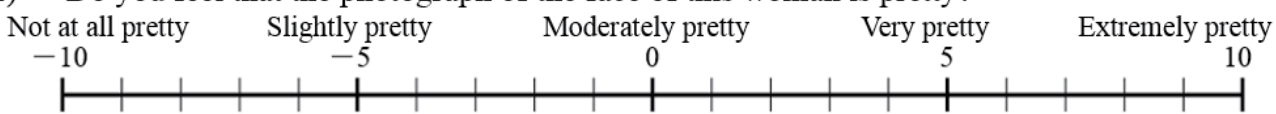

(3) Do you feel the photograph of the face of this woman is adult like?

Not at all adult like Slightly adult like Moderately adult like Very adult like Extremely adult like

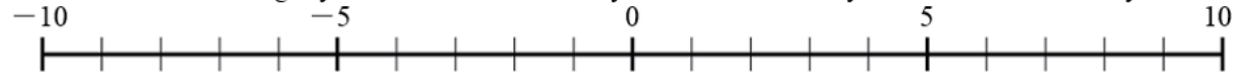

(4) Do you feel the photograph of the face of this woman has a bright expression?

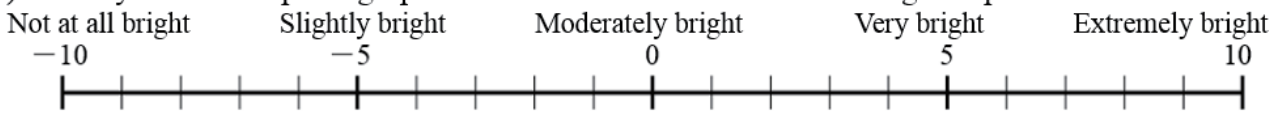

(5) Do you feel the photograph of the face of this woman is pleasant?
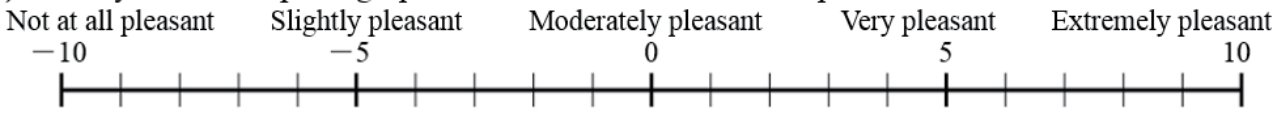

(6) Do you feel the photograph of the face of this woman is natural?

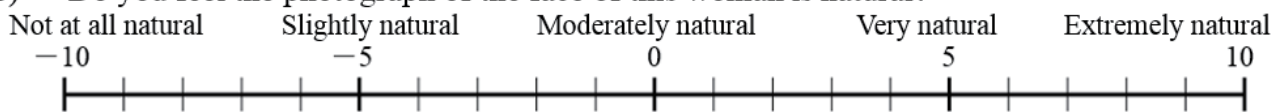

(7) Do you feel desire to look at the photograph of the face of this woman?

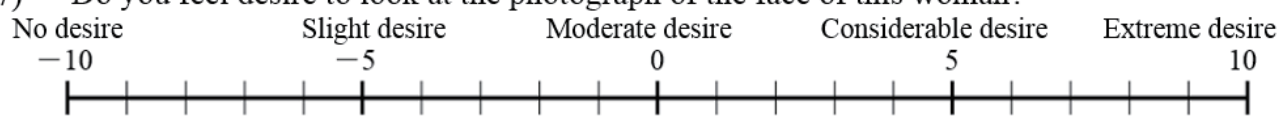

(8) How well do you know the woman in this photograph?

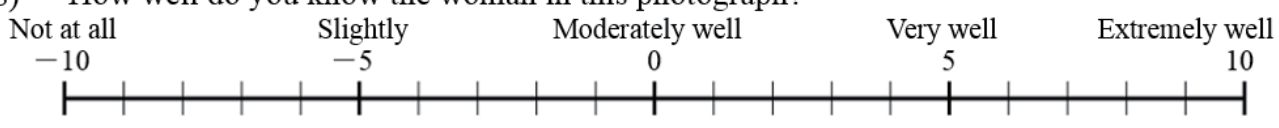

(9) What kind of expression does the woman in the photograph have?

Please check the appropriate answer.

( ) Happiness, ( ) surprise, ( ) sadness, ( ) anger, ( ) disgust, ( ) fear, ( ) expressionless

Fig. 1. (Color online) Questionnaire. 
(4) Electrode placement for EEG recording

Electrodes were placed in the following sites using 11 channels based on the international 10-20 system. We positioned the ground electrode on the forehead and reference electrodes on the right (A1) and left ears (A2) using referential derivation. Measurement sites included Fp1 and Fp2 (frontal pole), F3 and F4 (frontal), Fz (midline frontal), C3 and C4 (central), Cz (vertex), P3 and P4 (parietal), and $\mathrm{Pz}$ (midline parietal). The electrooculogram was also recorded to remove artifacts associated with blinking.

(5) Procedure

The experiment was conducted according to the flow diagram shown in Fig. 2. The nine words to be memorized were displayed. Then, a recognition stimulus word was presented, and the participants were asked to respond whether or not the word was included in the words to be memorized by pressing a key. After the recognition stimulus was presented, a 1.5-s interval was included for completing the response. If a participant pressed the key, there was an interval of just $1 \mathrm{~s}$ and then the display was switched to a feedback screen of either a female facial image (rated as highly attractive or moderately attractive) as a reward for a correct response or a gray image (no reward) for an incorrect response. The process from the display of the recognition stimulus to

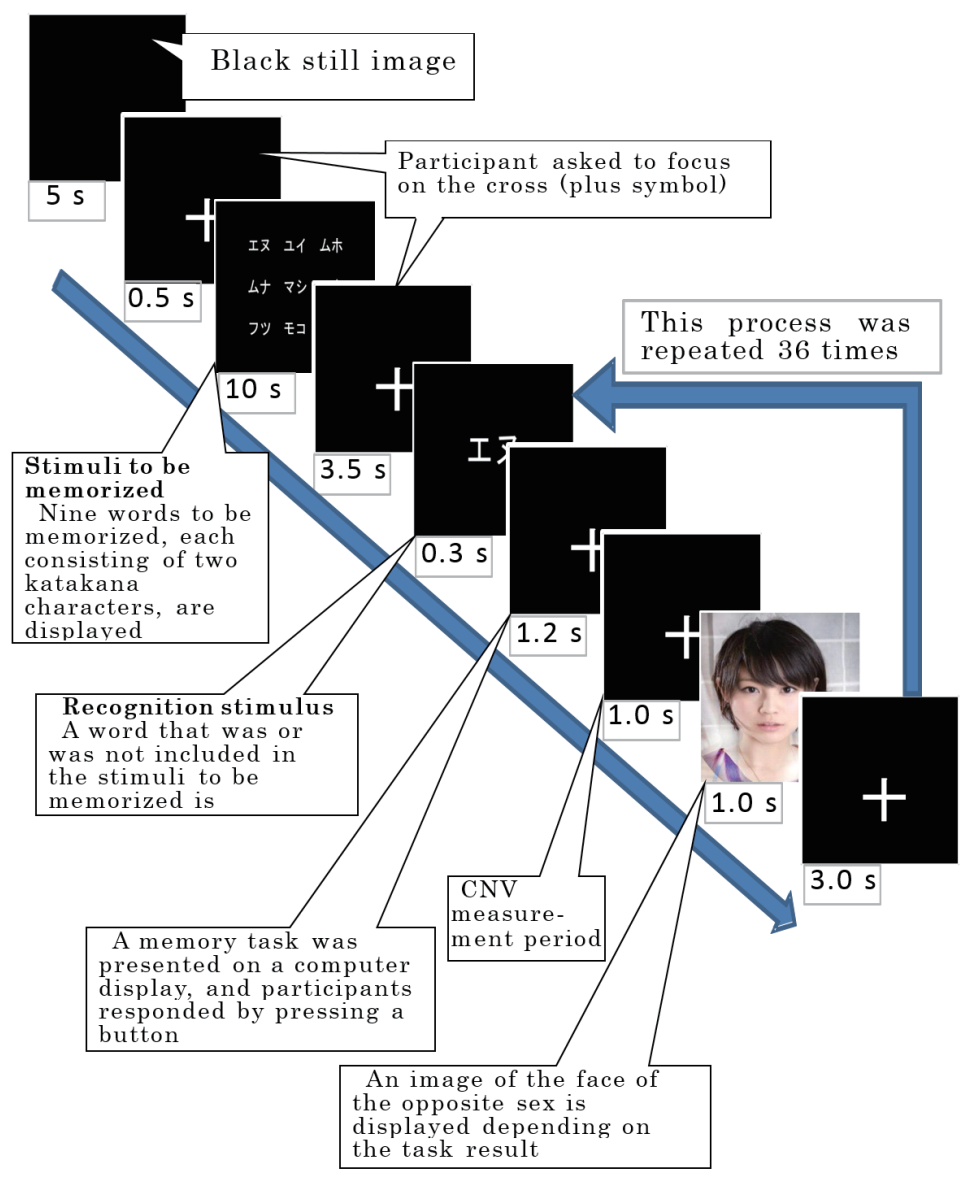

Fig. 2. (Color online) The task flow for one session. 
feedback was considered one trial, which was repeated 36 times to make up one session. In every session a participant was presented nine new words to memorize.

Of these 36 recognition stimuli, 12 words were those included in the stimuli to be memorized and the remaining 24 words were new. The 36 trials were divided into two halves, and the correct response rates for both halves were examined and compared. A total of six trials using highly attractive female facial images and six trials using moderately attractive female images as rewards were conducted. The images appeared as rewards randomly.

\section{Analysis}

\subsection{Analysis of EEG data}

EEG data between $-1500 \mathrm{~ms}$ (pre display of a reward stimulus) and $1000 \mathrm{~ms}$ (post display of a reward stimulus) were analyzed for the measurement sites listed in $\S 2.2$ (4).

\subsection{Analysis of CNV}

First, a 10th-order Butterworth band-pass filter $(0.5-30 \mathrm{~Hz})$ was applied to the brain wave data to remove high-frequency noise. Next, we removed trials that included artifacts associated with eye movements or blinking from the recorded EEG from the 11 channels by referring to the electrooculogram data. Only those trials without artifacts were averaged. We set the threshold for the electrooculogram potential as $100 \mu \mathrm{V}$ to remove artifacts.

Finally, the baseline value (the average value between $-1500 \mathrm{~ms}$ before the reward stimulus display and $1000 \mathrm{~ms}$ after the stimulus display) was subtracted from the average waveform described above to yield the ERP waveform. In this study, the maximum amplitude measured between $-1000 \mathrm{~ms}$ before the reward stimulus display and that after the display was regarded as the $\mathrm{CNV}$ value. $\mathrm{CNV}$ values were compared between images of different attractiveness.

\section{Results}

Figure 3 shows the averaged CNV values of the seven participants. The previous reward impacts the expectation effect of next reward. The results suggest that the CNV value was higher for reward images judged as more attractive than for reward images judged as moderately attractive.

As indicated in Fig. 4, the correct response rate was greater when a highly attractive image was presented than when a moderately attractive image was presented as a reward. The impact of reward type upon performance became apparent only after a number of sessions. We parsed the data from the first six and last six sessions of each participant.

In addition to presenting improved accuracy in response to highly attractive rewards over moderately attractive rewards, participants also presented improved performance when the first set of 6 sessions was compared to the last set of 6 sessions. Furthermore, the correct response rates for the first and second halves of a single session showed that accuracy was enhanced when a highly attractive image was used as a reward (see Fig. 4). 


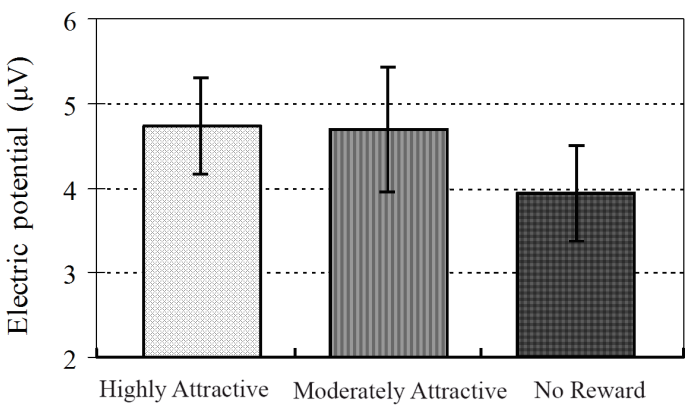

Fig. 3. Averaged CNV values of seven participants.

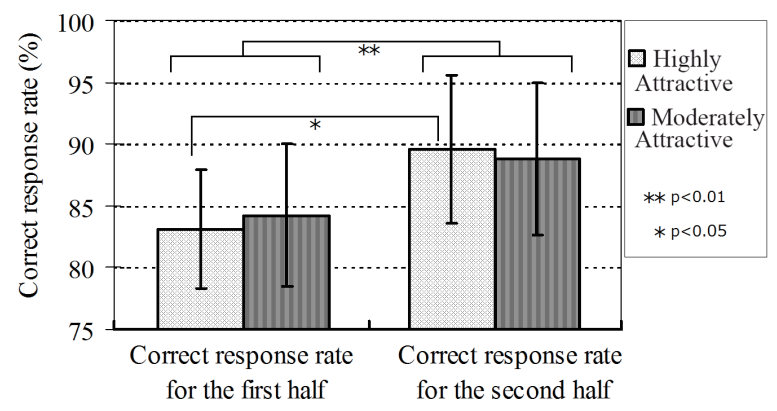

Fig. 4. Correct response rates for the first and second halves of one session.

\section{Conclusions}

The results of this experiment indicated that highly attractive female facial images used as rewards in a memory task evoked larger CNV values. This suggests that the more attractive the facial image, the greater the anticipation for the reward. In addition, the accuracy rate for the memory task improved when an attractive face was presented as the reward stimulus.

Our experiment was conducted with male participants and female images as rewards. Further experiments with female participants and male rewards would be desirable.

These results should also be confirmed using a larger sample size. In the future, we are planning to use $\mathrm{CNV}$ as a key parameter for developing BMIs to enhance cognitive functions.

\section{Acknowledgements}

This work was supported by KAKENHI (Grant-in-Aid for Scientific Research (C) Nos. 21610007 and 24600005, Grant-in-Aid for JSPS Fellows No. 11J40189, and Research Activity Start-up No. 70581180).

\section{References}

1 W. G. Walter, R. Cooper, V. J. Aldridge, and W. C. McCallum: Nature 203 (1964) 380

2 G. Kohls, J. Peltzer, B. Herpertz-Dahlmann, and K. Konrad: Dev. Sci. 12 (2009) 614.

3 K. Jimuraa, H. S. Lockea, and T. S. Braver: Proc. Nat. Acad. Sci. USA (PNAS) 107 (2010) 8871.

4 I. Aharon, N. Etcoff, D. Ariely, C. F. Chabris, E. O'Connor, and H. C. Breiter: Neuron 32 (2001) 537.

5 H. H. Jasper: Electroencephalography Clin. Neurophysiol. 10 (1958) 371.

6 R. T. Wilkinson and S. M. Susan: Biol. Psychol. 1 (1974) 167. 


\section{About the Authors}

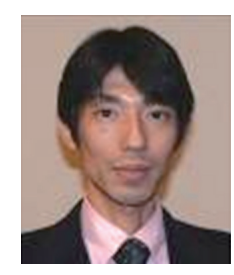

Yasuhiro Ogoshi is an Associate professor at the Graduate School of Engineering, University of Fukui in Japan. He received a Ph.D. Degree in Engineering from the University of Kanazawa in 2001. He is engaged in studies relating to human interfaces.

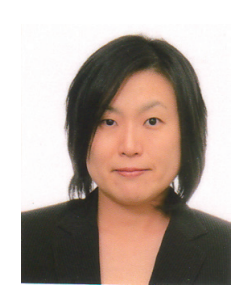

Sakiko Ogoshi is an Associate Professor at the Department of Electrical Engineering and Computer Science National Institute of Technology, Fukui College, in Japan. She received a Ph.D. Degree in Engineering from the University of Kanazawa in 2004. She was a Researcher at the Japan Society for the Promotion of Science and the Faculty of Education and Regional Studies, University of Fukui from 2011 to 2014. Her research interests include special needs and developmental disorders and knowledge management systems applied to information technology.

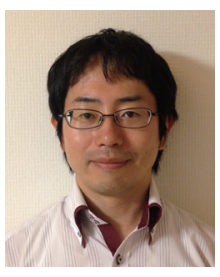

Tomohiro Takezawa was awarded a Ph.D. in Psychology from Hiroshima University. He specializes in studying cognitive abilities and assistive technology.

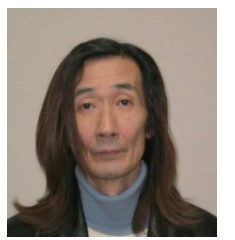

Yoshinori Mitsuhashi is a Professor Emeritus and currently a specially-assigned professor on the Faculty of Education and Regional Studies at Fukui University, Japan. After graduating from Kwansei-Gakuin University, Department of Experimental Psychology, in 1972, he finished his graduate studies in 1980 . He became an associate professor at Fukui University in 1981 and left as a professor in 2014. His research covers psychophysiology of cognition and education of handicapped children, especially studies using electrical acitivity of brain cognitive processes in children and adults with neurodevelopmental disorders. 